\title{
Approaches to mimic the metallic sheen in beetles
}

\author{
Lenau, Torben Anker; Aggerbeck, Martin; Nielsen, Steffen
}

Published in:

SPIE Optics \& Photonics

Link to article, DOI:

$10.1117 / 12.826227$

Publication date:

2009

Document Version

Peer reviewed version

Link back to DTU Orbit

Citation (APA):

Lenau, T. A., Aggerbeck, M., \& Nielsen, S. (2009). Approaches to mimic the metallic sheen in beetles. In SPIE Optics \& Photonics: The Biomimetics and Bioinspiration conference (Vol. 7401, pp. 740107). SPIE -

International Society for Optical Engineering. https://doi.org/10.1117/12.826227

\section{General rights}

Copyright and moral rights for the publications made accessible in the public portal are retained by the authors and/or other copyright owners and it is a condition of accessing publications that users recognise and abide by the legal requirements associated with these rights.

- Users may download and print one copy of any publication from the public portal for the purpose of private study or research.

- You may not further distribute the material or use it for any profit-making activity or commercial gain

- You may freely distribute the URL identifying the publication in the public portal

If you believe that this document breaches copyright please contact us providing details, and we will remove access to the work immediately and investigate your claim. 


\title{
Approaches to mimic the metallic sheen in beetles
}

\author{
Torben A. Lenau*a, Martin Aggerbeck ${ }^{\mathrm{a}}$, Steffen Nielsen ${ }^{\mathrm{a}^{*}}$ \\ ${ }^{a}$ Technical University of Denmark, DTU Management Building 424, DK2800 Lyngby, Denmark
}

\begin{abstract}
A range of different beetles exhibits brilliant colours and metallic sheen. One of the most spectacular species is the Plusiotis resplendens from Central America with gold metal appearance. The beetle shells are made from chitin and have a number of unique properties that apart from spectacular aesthetic effects include metal sheen from non-metal surfaces combined with electric and thermal insulation. The reflection mechanism has been studied by a number of authors and is well understood. Basically there are 2 different reflection principles. One is the multilayer reflector where alternating layers have high and low refractive index. The other is the Bouligand structure where birefringent chiral nanofibres are organised in spiral structures. The paper describes work done to explore different approaches to mimic these structures using polymer based materials and production methods that are suitable for more complex double curved geometry. One approach is to use alternating layers of 2 different polymers applied by dipping and another is applying cholesteric liquid crystals in paint. However, none of them can yet make the desired metal-looking free-form surfaces.
\end{abstract}

Keywords: Biomimetics, aesthetics, beetles, structural colour, thin layer interference, bouligand structure, cholesteric liquid crystals.

\section{INTRODUCTION}

The present paper describes work done in order to map the state-of-art within using structural colours to achieve aesthetic pleasing surfaces using materials and technology with a mass production potential. Inspiration comes from nature where in particular many beetles have remarkable bright colours and metallic appearances.

Aesthetic quality is of course a very subjective matter and depends highly on the way it is used in a specific product. However, some material properties are often used in order to produce aesthetic pleasing products. They include factors that we sense with our eyes like

- bright saturated colours,

- metallic sheen (bling),

- angle dependent colours (iridescence),

- colour change based on environmental input (like temperature),

- colour change on demand and

- patterns (colour differences on the same surface).

But the aesthetic comprehension of our surroundings also depends on what we register with our other senses. For instance is part of the aesthetic impression of a chair that it is soft to sit in and gives a nice warm feeling. Nanostructured polymer surfaces has the potential of looking like metal but at the same time being insulating thermally (warm feeling on touch) and electrically (no risk of electric shock).

Metal looking polymer surfaces is therefore interesting for a long range of consumer products like mobile phones, bicycle helmets, children's toys, furniture, cars, jewellery, shoes and clothes.

\footnotetext{
*lenau@dtu.dk; phone +45 4525 4811; fax +45 4593 3435; www.dtu.dk; www.polynet.dk/lenau
}

Cite reference: Lenau, T. A., Aggerbeck, M., \& Nielsen, S. (2009). Approaches to mimic the metallic sheen in beetles. Spie Optics Photonics, 7401(1), 740107. doi:10.1117/12.826227 

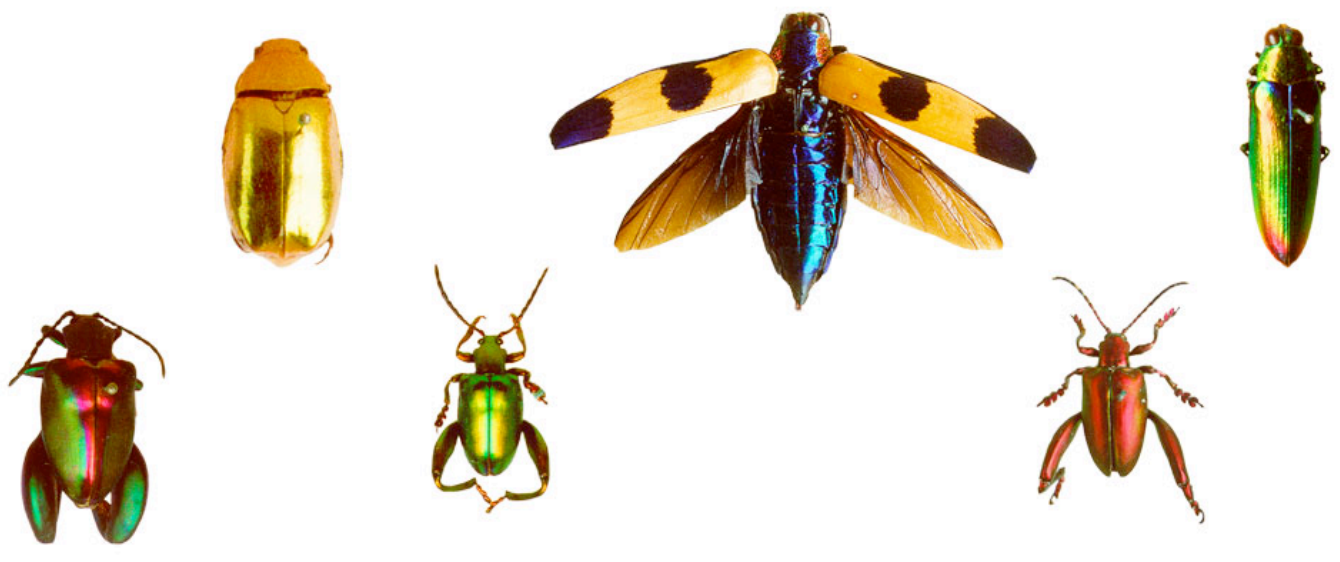

Fig. 1. Different beetles with metallic sheen. The golden Plusiotis resplendens scarabae is shown at the top left.

The effects are seen in nature and are mainly based on the structural colour principle, where white light interfere constructively and destructively in photonic crystals. There are many principles to achieve special optical effects like described by Srinivasarao [1]. Focus for the present works is however on thin layer interference and on the so-called Bouligand-structures as described by Lenau \& Barfoed [2], in both cases structures where white light interfere due to differences in refractive indices in photonic crystals in thin layers of thin fibres. The interference is recognised by the iridescence where the colour changes towards shorter wavelength for more grazing incidence or viewing angle.

\section{THIN LAYER INTERFERENCE}

Basically, thin layer interference can be made in quarter wave stacks, where layers of alternating high and low refractive index are placed on top of each other. When the layers have an optical thickness (refractive index $n$ multiplied with layer thickness $d$ ) equivalent to a $1 / 4$ wavelength they reflect the matching wavelength when illuminated by white light. The remaining light is transmitted to layers below or absorbed. For an ideal stack very high reflection is reached for only 10 20 layers for a selected wavelength.

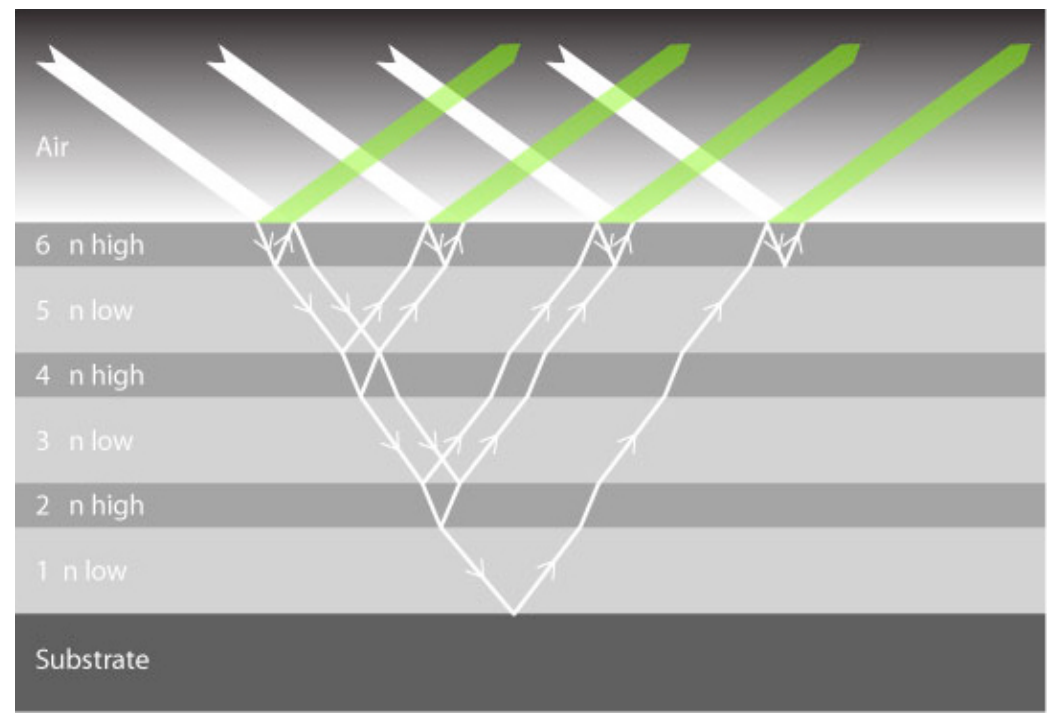

Fig.2. Thin layer interference. In an ideal quarter wave stack an even number of thin transparent layers reflect light that interfere. 


\subsection{Existing thin layer solutions}

The most basic version of thin layer interference is a single layer. This is widely used for coating of lenses and is often achieved by physical vacuum deposition of a high refractive index material onto the substrate. Polymer substrates can be coated and metallic appearance is for instance seen in some sunglasses. Even though bulk application exist it is not an inexpensive method. Besides the iridescence is very apparent.

Single layer coatings for structural colours on metal is described by Kikuti [3]. It is a electrochemical process that introduce an oxide layer to the metal surface. Similar methods are used for decorating household appliances in Japan and metallic façade elements in China.

Weber and colleagues report on so-called giant birefringent optics in multilayer polymer mirrors. They have made a very high reflective polymer film consisting of several hundred layers of PMMA and birefringent polyester. Reflectivity is close to $100 \%$ for wavelengths between $400 \mathrm{~nm}$ and close to $1 \mu \mathrm{m}$ [4].

Teijin Fibres has recently put the Morphotex textile fibre on the market. It is a light-reflective fibre inspired by the morpho-butterflies. Each fibre has a diameter of about 15-17 $\mu \mathrm{m}$ and holds a multilayer reflector with 61 layers of polyester and polyamid [5].

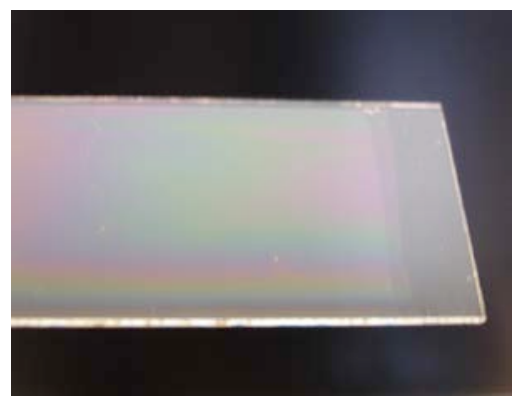

Fig. 3. A glass sample with a homogeneous layer of PVAC. The layer has been removed a right with a knife to allow for thickness measurement.

\subsection{Thin layer reflectance through dipping}

Most of the existing applications of thin layer interference using polymer materials only apply to flat geometries. We have conveyed an experiment to test the hypothesis that a multilayer polymer coating can be made by simply dipping the object in the 2 different polymer materials. To make the polymers fluent they were dissolved in a solvent. A range of different polymers and solvents were tried. The most successful ones were PS dissolved in THF (tetrahydroforan) and PVAC (polyvinylacetate) dissolved in acetone. The refractive index for PS is 1,59 and for PVAC it is 1,47. The solvent are very volatile causing very uneven coatings, but we succeed in achieving uniform layer thicknesses by carrying out the dipping in closed containers where the air was saturated with solvent. In this way only the surface tension forces were important for the layer thickness. Our goal was to make an ideal quarter wave stack, and this meant that the desired layer thicknesses were $86 \mathrm{~nm}$ for PS and $94 \mathrm{~nm}$ for PVAC. We used ordinary microscope glasses as substrate. By changing the concentration of the polymer in the solvent we managed to control the layer thickness and also to get visible colour reflections, even though they were rather week, see figure 3. Layer thickness were measured using a Nanoscan AFM Microscope. We did not succeed to apply more than two layers. Even though the polymers only dissolved in the one solvent, did the solvents used for layer 3 affect the under laying layers - they cracked and made small bubbles. Our conclusion is that it is possible to apply uniform layers of the right thickness using dipping. The challenge is to find a suitable combination of polymers and solvents for more layers.

\section{HELICAL BOULIGAND STRUCTURES}

Around 1970 the French zoologist Yves Bouligand described helical structures found in the exoscelleton in many arthropods. These structures are referred to as Bouligand structures [6,7]. Bouligand structures with optical reflection properties are helical arrangements of chiral transparent fibres (or crystals) of a birefringent compound, i.e. the refractive index is different in the direction of the long axis (called the director) and the directions perpendicular to the director. Due to the chiral property the fibres align with a twist forming a helical structure as illustrated in figure 4 . The fibres orient themselves with the director parallel to the surface. Incident light is partly reflected and partly transmitted. The 
optical properties in Bouligand structures are very similar to those found in cholesteric liquid crystals (CLC); however a major difference is that they are solid. For both structures the reflected part of the light is circular polarized in either a left-handed or a right-handed direction, which means that a reflector with a single handedness only reflect up to half the light [8]. For effective reflectors a high reflection can be achieved with as little as 20-40 pitches, which for green light gives a layer thickness of 10-20 microns [8] Combining 2 reflectors with different handedness makes higher reflections possible as illustrated in figure 7 .

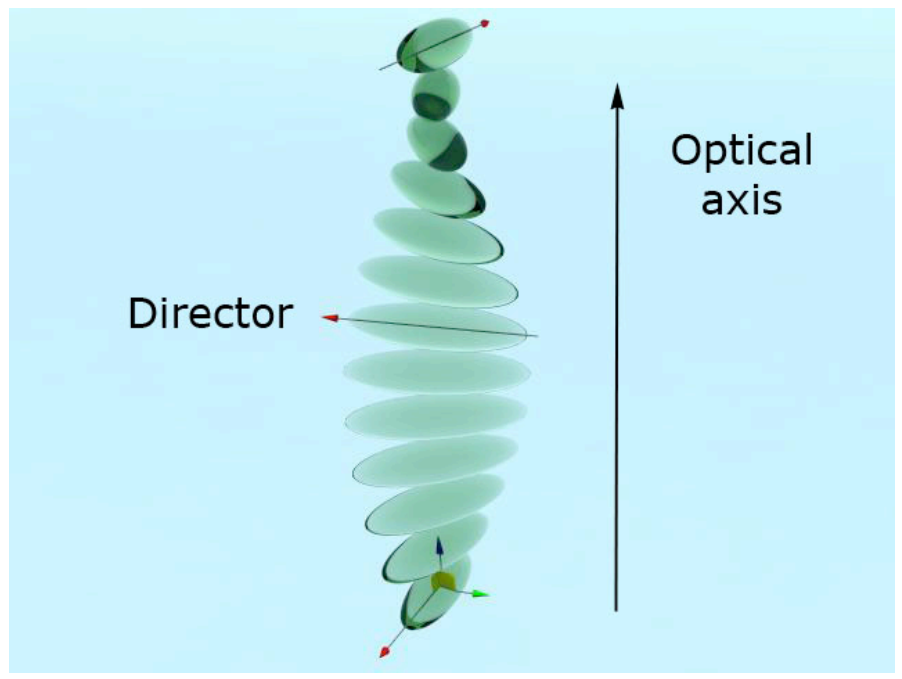

Fig. 4. Bouligand structures are helical arrangements of chiral transparent fibres (or crystals) of a birefringent compound. They remind of cholesteric liquid crystals except they are solid.

The Bouligand-structure in beetles is a solid, but the optical properties remind of those in cholesteric liquid crystals (CLC), also called twisted (or chiral) nematic liquid crystals. We will here look at CLC to find out if they can be used for surface coating purposes where aesthetic pleasing properties are desired by answering the five questions below:

- To what extend can bright colours and colour change be made with CLC?

- How can the properties be controlled?

- Is metallic appearance as seen in beetles possible?

- Can iridescence be controlled?

- Can the liquid crystal structures be made solid?

\subsection{Colours and colour change in CLC coatings}

We will answer this question by looking at existing applications, by searching the literature and through experiments.

On the market there is a number of commercial products claiming to use CLC. They include products like thermometers, electronic diagnostic paints, mood rings, coffee cups, and a nail polisher $[9,10,11]$ shown in figure 5 . The first three products change colour depending on the temperature. Basically they are red at a low temperature changing over yellow, green to blue as the temperature rises. This is opposite to what one would intuitively expect - that the heat expansion would lead to a larger pitch and therefore a longer reflected wavelength. But in the liquid crystal phase the CLC-crystals can rotate as temperatures rises causing a smaller pitch. The coffee cup and the nail polisher do not change their colour as temperature varies. In the Hallcrest handbook [12] the 2 types of CLC are called temperature-sensitive and temperature-insensitive.

CLC's are generally viscous compounds that are sensitive to the atmospheric air and to UV-light. For practical applications they need to be sealed from the environment. One method is to use microcapsules - tiny polymer spheres that hold the CLC [12]. This method is used in the products mentioned above. 

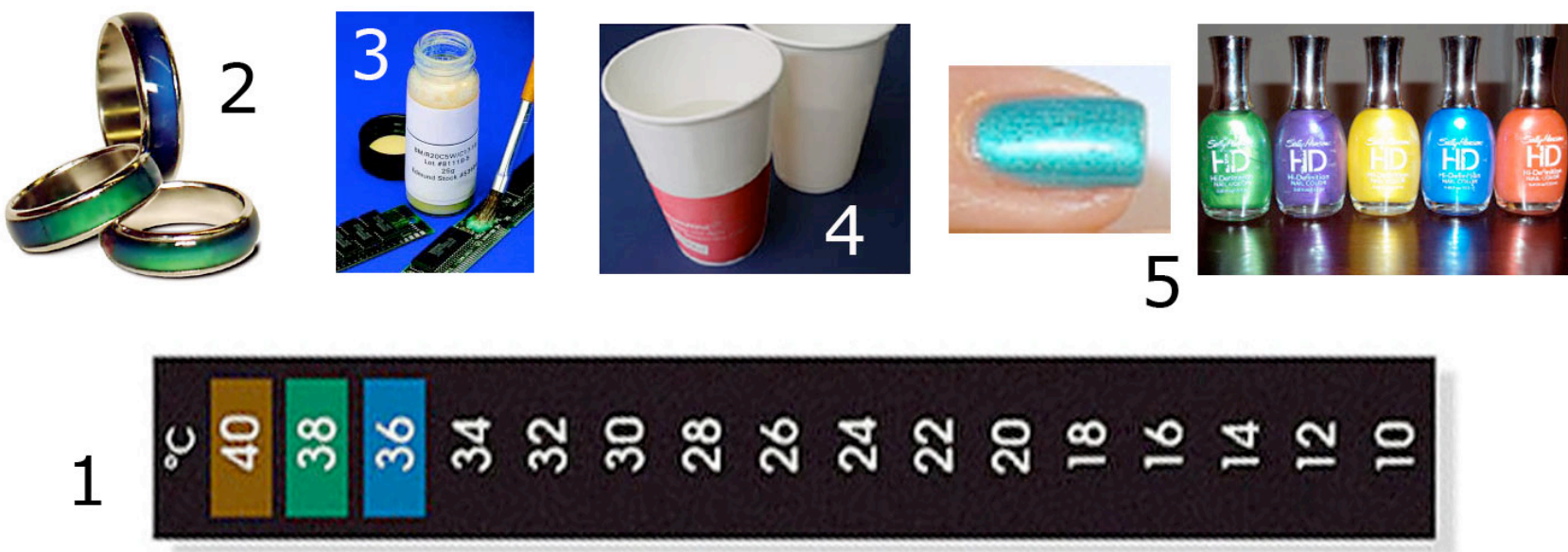

Fig. 5. Commercial products using cholesteric liquid crystals: 1. Thermometer, 2. Mood rings, 3. thermal liquid crystal paint, 4. temperature sensitive coffee cup and 5. Nail polish.

In the literature there are large amounts of papers on CLC for various purposes, many of them focus on displays, optical memory and data transfer.

Tamaoki and colleagues reports on a cholesteryl-based temperature-sensitive CLC where the colour change takes place over a temperature range from 87-115 degree Celsius [13,14]. By quenching the compound in ice water from a certain temperature the 'frozen' solid can keep its colour. The process is reversible and in principle this technique can be used for rewritable memory and for customizable surface colours including patterns. Transmitted light is reduced from over $80 \%$ to over $40 \%$ for the affected wavelength. This indicates a fairly high reflectance.

Another question is which chemicals that are used in CLC. Makov used a left-handed CLC mixed from cholesteryl nonanoate, cholesteryl chloride and cholesteryl oleyl carbonate and a right-handed CLC consisting of an active amyl alcohol derivative [8]. Hallcrest distinguishes between cholesteric and chiral nematic materials [12]. They behave similarly, but the first is based on cholesterol and other sterol derivatives. The chiral nematics comprises of non-stereol based chemicals.

We have experimented with 3 types of CLC: A premixed CLC Merck Licristal 30823, MDA-00-1445 with a blue-green colour and a nematic LC Merck MLC 6608 that we doped with either a left- or a right-handed dopant (MLC 6247 and 6248 ) to get CLC. The birefringence for 6608 is $\Delta \mathrm{n}=0.083$ at $\lambda=589 \mathrm{~nm}$ [15]. The compounds were positioned between two glass plates and measurements were made using a halogen light source and an Eliott photospectrometer. By changing the dopants we obtained different reflected colours ranging from red to yellow, green and blue, see figure 6 . The width of the reflected spectra were around $60 \mathrm{~nm}$ and intensity of the light was from $25-35 \%$. The CLC is closest to the temperature-insensitive type. When heating a CLC-sample from 20 to 90 degree Celsius the shape of the reflected wavelength curve only changed slightly. The intensity of the reflected light decreased until the phase change temperature where the sample became transparent. 


\section{Amount of dopant in wt\%, 45.0 degrees}
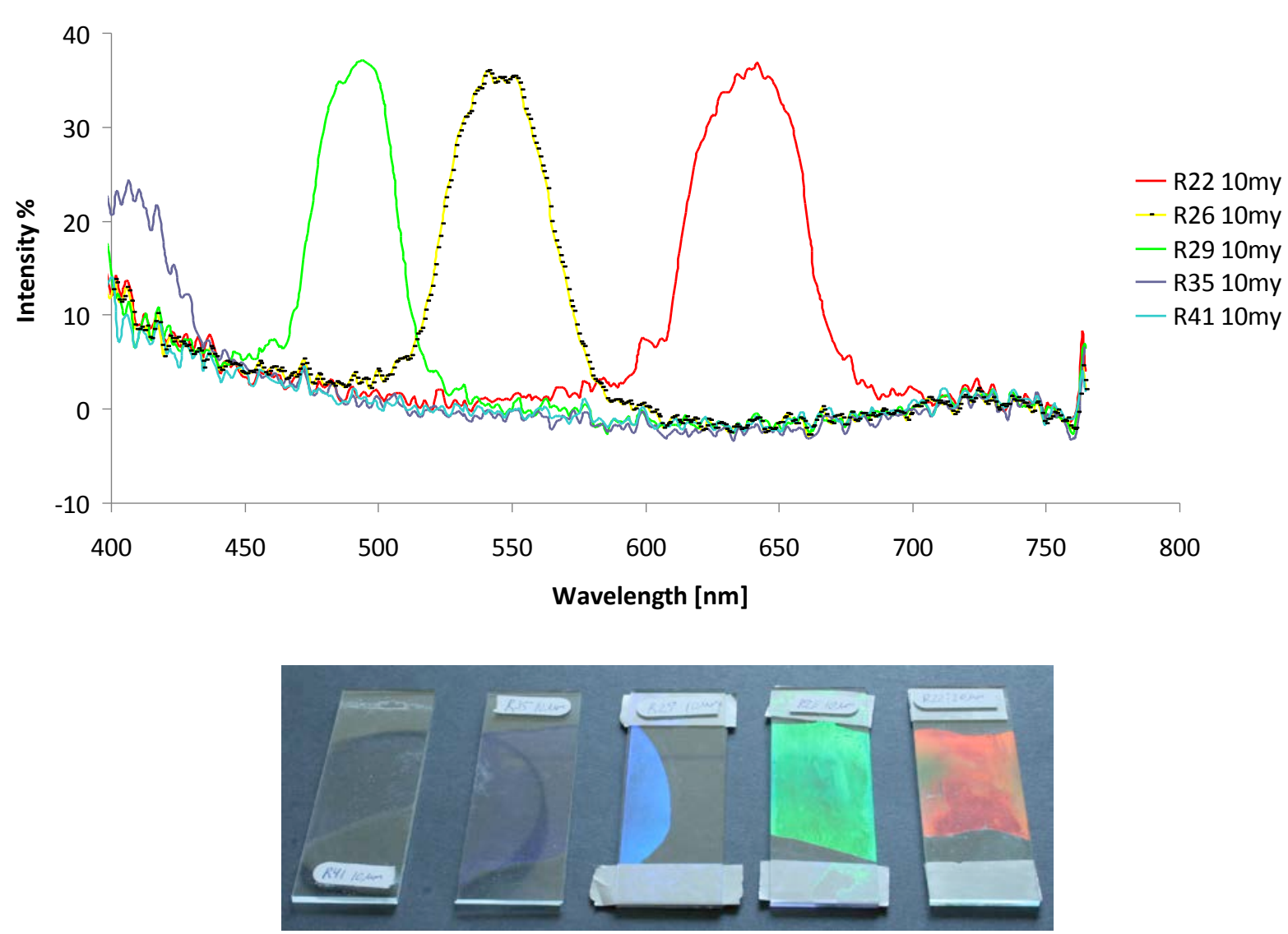

Fig. 6. Reflectance spectra at 45 degree incidence and observation angle for Merck MLC 6608 doped with (from right to left) $22 \%$ (red curve), 26\% (yellow curve with black spots), 29\% (green curve) and 35\% (blue curve) Merck MLC 6248. Measurements are normalised regarding light source and glass reflections. The picture shows slightly 'colder' colours due to a different angle of incidence.

Therefore the answer to the question is that CLC applied to surfaces can be made in many different colours and with fairly high intensity of the reflected light. Some CLC change colour depending on the temperature while others keep a fixed colour independent of the temperature within a certain interval. The colour for the first type can be tuned by quenching, and patterns can be made as far as temperature differences can be controlled across the surface (e.g. using lasers).

\subsection{Controlling the properties in CLC coatings}

Up to $50 \%$ reflectivity is theoretically possible for reflection of single handed polarized light. Srinivasarao reports reflections about 40\%. [1] Makow superimposes a left and right handed CLC and measures $85 \%$ reflectance [8]. Jeong and colleagues report that they have combined two layers of different handed CLC achieving more than $90 \%$ reflectivity [16]. In our own experiments we also tried to superimpose a right and left handed CLC as shown in figure 7. The reflections from the samples seems larger from where the 2 samples overlap. Measurements of the samples in figure 7B confirms that the combination of left and right handed CLC reduces the transmittance compared to the 2 layers of left handed CLC, i.e. they have a higher reflectance. 
As described above a wide range of colours can be made. Basically the colour is dependant of the pitch, which again is controlled by the chemical composition - the amount of dopant - a higher amount of dopant shifts the colour towards shorter wavelength. Temperature is also influential, and higher temperature normally causes a shift towards shorter wavelengths.

A
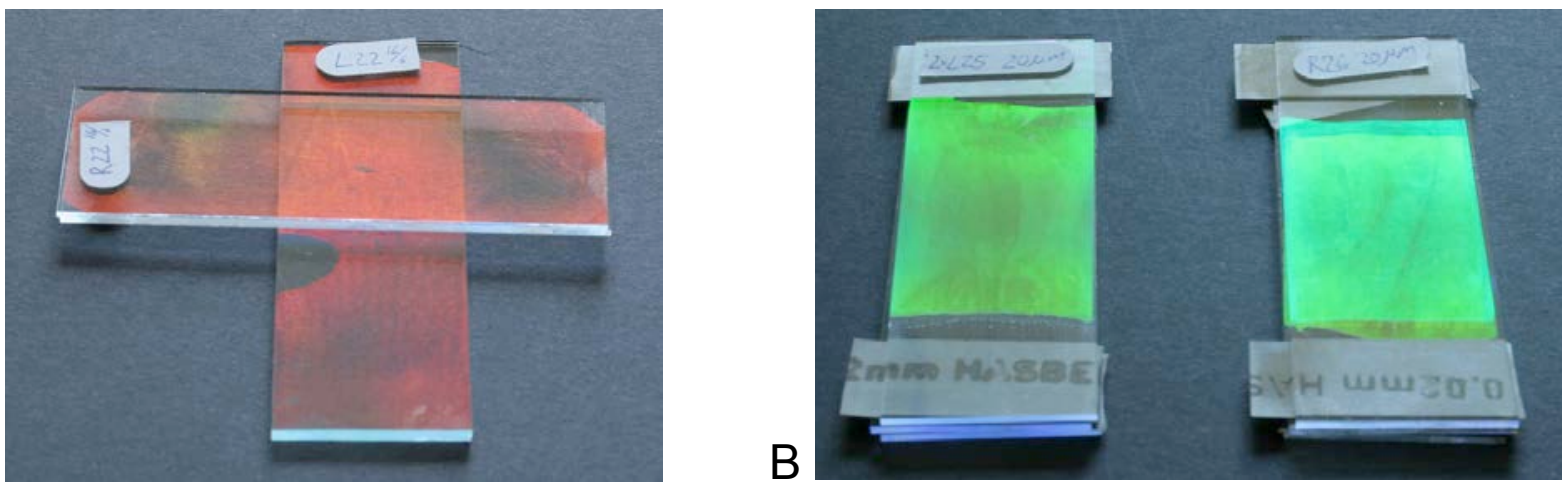

Fig. 7. A) A left-handed and a right handed CLC placed between glass-plates. Apparently the reflection looks larger where the 2 samples are superimposed. Samples have 22\% dopant. B) The samples are placed between three glasses. The left one has $2 \times 25 \%$ left handed dopant and the one to the right which looks brighter has $25 \%$ left handed and $26 \%$ right handed dopant.

\subsection{Metallic sheen and width of spectrum}

Colourless metallic sheen require that the whole visual spectrum is reflected. The question is if this can be done with CLC. Most papers and our own measurements indicate that a CLC has a fairly low bandwidth - typically less than 100 $\mathrm{nm}$. Mitov describes the relationship between bandwith $\Delta \lambda$ and birefringence $\Delta \mathrm{n}$ (the difference between the ordinary and the extraordinary refractive index) as $\Delta \lambda=\mathrm{p} \Delta \mathrm{n}$ where $\mathrm{p}$ is the pitch [17]. $\Delta \mathrm{n}$ is normally limited to 0,3 for colourless organic materials which set the limit to $100 \mathrm{~nm}$ for $\Delta \lambda$ [17].

The solution to more broadband and metallic reflection could be to superimpose more than one CLC in order to cover the visual spectrum. Mitov and colleagues report to have made a broadband reflector with a metallic aspect. They use an oligomer type CLC and makes two glass plates; one doped to become blue $\lambda=445 \mathrm{~nm}$ and another to become red $\lambda=710 \mathrm{~nm}$. A sandwich cell is made with $20 \mu \mathrm{m}$ spacers, the sample is heated to 85 degrees causing a diffusion and then quenched to room temperature. In this way the helix structure is maintained. The result is about $50 \%$ reflection over most of the visible spectrum causing a colourless metallic appearance. [17]

\subsection{Iridescence and angle dependency}

The iridescence or angle dependency is an inherent property of the CLC. Hallcrest describes that the reflected wavelength can be estimated by multiplying the pitch with the mean refractive index and $\sin (\theta)$, where $\theta$ is the angle between incidence and observation positions [12].

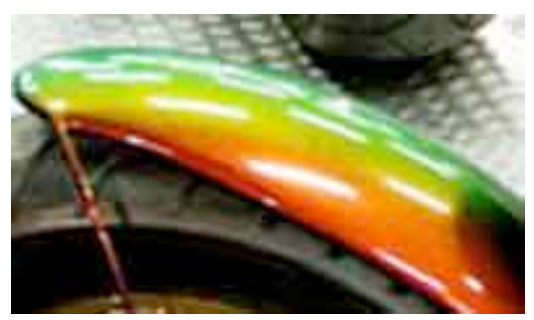

Fig. 8. A colour change paint

In our experiments the iridescence was in the order of $100 \mathrm{~nm}$ when the angle was changed from 22,5 to 67,5 degrees. 
The spectra FX paint from Alsapaint has an colour change that seen from pictures in figure 8 is twice as large - about $200 \mathrm{~nm}$ for 45 degrees. The company describes the paint as based on crystal flakes, but it is not clear if they mean CLC [18]. There are however patents for the use of CLC in the form of flakes in paints [19, 20, 21]

\subsection{Liquid or solid?}

The viscous characteristic in CLC is an advantage when movement of the crystals are desired, e.g. for temperature sensors. But surfaces in contact with humans should be wear resistant and must not stick to the fingers. Different methods for 'freezing' or curing the CLC can therefore be applied.

One method is quenching, i.e. quick cooling from a higher temperature to a solid state at room temperature. This is reported by Palffy-Muhoray and by Tamaoki. They describe how a CLC that is cholesteric in the temperature range $87-$ 115 degree Celsius can be heated and quenched resulting in a range of different colours. The material can be used for rewritable full colour media $[13,14]$.

Another method is described by Zapotocky and colleagues. They describe a method where colloidal particles with a size of about 1 micron can be used to stabilize the liquid CLC so it behaves like a solid [22].

\section{METAL LOOKING POLYMER SURFACES}

Nature shows many examples of brightly looking metal appearances in beetles. Insects are made from chitin and protein materials that have polymer characteristics. A biomimetic approach to obtain a metallic look is therefore to make artificial polymer surfaces with similar properties. Metal looking polymer surfaces are allready made for some, especially flat, geometries and are based on both thin layer interference and helical Bouligand structures.

Thin layer interference is applied in a number of applications and can be used to make a broad range of bright saturated colours. Metallic sheen and angle dependent colours (iridescence) can also be achieved and is often a key reason for their application. Colour change based on environmental input (like temperature) and colour change on demand do not seem to be made. However the later is seen in nature where the Apistomorpha tecta beetle change colour from gold to red when in danger [23]. Patterns in the form of colour differences on the same surface has not been made by directly controlling the optical structure. In our experiments we have succeeded in achieving uniform layers of polymers by dipping, but we have not solved the problem of applying more than 2 layers in a multi layer stack.

Helical Bouligand structures in beetles have the fascinating and attractive characteristic of organizing automatically through self-assembly. Since cholesteric liquid crystals (CLC) behave similarly to the Bouligand structures from an optical point of view we have researched different applications and conducted experiments to evaluate the use of CLC as surface coatings. CLC applied in surfaces can produce many different colours with a high light intensity. Tunable colours and patterns can be made using the temperature sensitive CLC's that are fixated through quenching. Another type of CLC reflects a single fixed colour independent of the temperature. CLC's normally have a narrowband reflection seen as a single colour, but there are techniques for mixing more CLC's to achieve broadband and metallic reflectance.

\section{ACKNOWLEGDEMENT}

Thanks to Siren Sundby and Jacob Telcs for performing the dipping experiment, and Sokol Ndoni from DTU Nanotech for support, discussions and supply of polymers for dipping. Thanks also goes to Ann Marie Dubgaard Andersson and Lars Georg Kiørboe from DTU Kemiteknik for materials and use of the Hempel Innovation Chemical Laboratory. We are indebted to Lara Scolari from DTU Fotonik and Sebastian Gauza from Creol, The University of Central Florida for valuable discussions and for supplying us with the CLC materials. Also thanks to Tomas Benzon for graphical work on the illustrations.

\section{REFERENCES}

[1] Srinivasarao, M., "Nano-Optics in the Biological World: Beetles, Butterflies, Birds, and Moths", Chem. Rev 99, pp.1935-1961 (1999) 
[2] Lenau, T. and Barfoed, M., "Colours and metallic sheen in beetle shells- a biomimetic search for material structuring principles causing light interference", Journal of Advanced Engineering Materials 10(4), 299-314 (2008).

[3] Kikuti, E., Conrrado, R., Bocchi,, N., Biaggio, S.R. and Rocha-Filho, R.C., "Chemical and Electrochemical Coloration of Stainless Steel and Pitting Corrosion Resistance Studies", J. Braz. Chem. Soc. 15(4), 472-480 (2004).

[4] Weber M.F.; Stover, C.A.; Gilbert, L.R.; Nevitt, T.J.; Ouderkirk, A.J., "Giant Birefringent Optics in Multilayer Polymer Mirrors", Science 287, 2451-2456 (2000)

[5] Morphotex, http://www.teijinfiber.com/english/products/specifics/morphotex.html

[6] Bouligand, Y., [Les arrangements fibrillaires torsades dans les matériels biologiques et leur signification], Doctoral thesis, Université de Paris (1971)

[7] Neville, A.C., [Biology of the Arthropod Cuticle], Springer-Verlag, Berlin Heidelberg New York, (1975)

[8] Makow, D.M., "Peak reflectance and color gamut of superimposed leftand right-handed cholesteric liquid crystals", Applied Optics 19(8), 1274-1277 (1980)

[9] LCR Hallcrest, http://www.davisliquidcrystals.com/

[10] Thermal liquid crystal paints, http://scientificsonline.com

[11] Sally Hansen, http://www.sallyhansen.com

[12] Hallcrest Handbook of Thermocromic Liquid Crystal Technology, http://www.davisliquidcrystals.com/downloads/randtk_TLC_Handbook.pdf

[13] Palffy-Muhoray, P., "New designs in cholesteric colour", Nature 391, 745-747 (1998)

[14] Tamaoki, N., Parfenov, A. V., Masaki, A. and Matsuda, H., "Rewritable full-color recording on a thin solid film on a thin colesteric low-molecular-weight compound", H. Adv. Mater. 9, 1102-1105 (1997).

[15] Rao, L., Gauza, S., and Wu, S.-T., "Low temperature effects on the response time of liquid crystal displays", Appl. Phys. Lett. 94, 071112 (2009)

[16] Jeong, S.M., Sonoyama, K., Takanishi, Y., Ishikawa, K., Takezoe, H., Nishimura, S., Suzaki, G. and Song, M.H. "Optical cavity with a double-layered CLC mirror and its prospective application to solid state laser", Appl. Phys. Lett. 89, 241116 (2006)

[17] Mitov, M. A. Boudet, A. and Sopéna, P., "From selective to wide-band light reflection: a simple thermal diffusion in a glassy cholesteric liquid crystal", Eur. Phys.J.B. 8, 327-330 (1999)

[18] Alsa Corporation, http://www.alsapaint.com/products/spectraFX.html

[19] Optical bodies containing cholesteric liquid crystal material and methods of manufacture, Patent no. AU2002228669 (2003)

[20] Effect lacquer and lacquering, in particular for car bodies, by means of liquid crystal Interference pigments, Patent No. WO9532247 (1995)

[21] Coatings with a cholesteric effect and method for the production thereof, Patent no. WO/1999/011733 (1999)

[22] Zapotocky, M., Ramos, L., Poulin, P., Lubensky, T. C. and Weitz, D. A., "Particle-Stabilized Defect Gel in Cholesteric Liquid Crystals", Science 283, 209-212 (1999)

[23] Hinton, H.E., "Some recent works on the colours of insects and their likely significance", Proc. Br. ent. nat. Hist. Soc. 6, 43 (1973) 